\title{
Piezogyration effect in paratellurite crystals
}

Mys O., Vasylkiv Yu., Martynyuk-Lototska I. and Vlokh R.

Institute of Physical Optics, 23 Dragomanov St., 79005 Lviv, Ukraine, e-mail: vlokh@ifo.lviv.ua

Received: 29.10 .2007

\begin{abstract}
In this work we present the results of experimental study of piezogyration effect in $\mathrm{TeO}_{2}$ crystals. Critical analysis of the conditions for piezogyration measurements and the corresponding data is performed. The value of the piezogyration coefficient $\beta_{3333}$ for the mentioned crystals is determined.
\end{abstract}

Keywords: $\mathrm{TeO}_{2}$ crystals, piezogyration

PACS: 78.20.Ek, 78.20.Hp

\section{Introduction}

Piezogyration effect known also as elastogyration manifests itself as a change of optical activity of a medium, in particular of a crystal, under the action of mechanical stress or deformation. Contrary to elastooptic effect, the piezogyration can exist only in noncentrosymmetric media. The relation for optical-frequency dielectric permittivity $\varepsilon_{i j}$ in the presence of piezo- and elastogyration may be written as

$$
\begin{aligned}
\varepsilon_{i j} & =\varepsilon_{i j}^{0}+i e_{i j l} g_{l k} k_{k}=\varepsilon_{i j}^{0}+i e_{i j l}\left(g_{l k}+\Delta g_{l k}\right) k_{k}=\varepsilon_{i j}^{0}+i e_{i j l}\left(g_{l k}+\beta_{l k m n} \sigma_{m n}\right) k_{k}, \\
& =\varepsilon_{i j}^{0}+i e_{i j l}\left(g_{l k}+\beta_{l k m n} C_{m n r t} X_{r t}\right) k_{k}=\varepsilon_{i j}^{0}+i e_{i j l}\left(g_{l k}+\delta_{l k t} X_{r t}\right) k_{k}
\end{aligned}
$$

where $\delta_{l k r t}=\beta_{l k m n} C_{m m r t}$ is the fourth-rank axial elastogyration tensor defined in the units of reciprocal mechanical deformation $X_{r t}, \beta_{l k m n}$ the fourth-rank axial piezogyration tensor defined in the units of reciprocal mechanical stress $\sigma_{m n}, C_{m n r t}$ the tensor of elastic stiffness coefficients, $k_{k}$ the wave vector, $g_{l k}$ the second-rank axial gyration tensor and $e_{i j l}$ completely antisymmetric Levi-Civita unit axial tensor. Thus, we write the increment of gyration tensor due to the piezo- and elastogyration effects as

$$
\Delta g_{l k}=\beta_{l k m n} \sigma_{m n},
$$

or

$$
\Delta g_{l k}=\delta_{l k r t} X_{r t} .
$$

Probably, the first definition of piezogyration effect has been made in 1964 [1] by 
K. Aizu as foolows: "The 'piezogyration' tensor means the tensor equal to the rate of change of the gyration tensor with the stress tensor at zero value of the stress tensor". The fourth-rank axial tensors in that paper have been derived only for the polar point groups of symmetry. The fourth-rank axial tensors that describe both the piezogyration and the quadratic electrogyration effects for all of the point symmetry groups, which permit existence of these effects, have been derived in 1970 by O. G. Vlokh and T. D. Krushel'nitskaya [2]. The analysis of the piezogyration effect for crystals belonging to a number of symmetry groups has also been reported in [3].

Experimental investigations of piezogyration under variations of hydrostatic pressure have been performed for the quartz and sodium chlorate crystals [4, 5]. However, hydrostatic pressure is a scalar action and so the manifestations of tensorial properties of the piezogyration could not be revealed in the investigation $[4,5]$. Later on, the piezogyration has been studied in the quartz crystals under application of mechanical stress component $\sigma_{33}$ [6]. The coefficient of piezogyration tensor has been determined in [6] as being equal to $\beta_{3333}= \pm(1.5 \pm 0.3) \times 10^{-12} \mathrm{~m}^{2} \mathrm{~N}^{-1}$ (with the signs " \pm " corresponding respectively to levorotary and dextrorotary quartz crystals).

It is necessary to note that the observed piezogyration effect is commonly very small and, probably, this is a reason of poor literature data related to the studies of that effect. Moreover, the experimental conditions for studies of the piezogyration are quite complicated. This is because the studies of this effect on the basis of direct measurements of polarization plane rotation, i.e. without inducing of accompanying linear piezooptic birefringence, require providing the application of longitudinal stress along the optic axis in optically uniaxial crystals (or along $<111>$ direction in some of cubic crystals).

However, there exists another condition for investigation of the piezogyration in crystals. For example, the measurements of the piezogyration effect in GaAs, ZnSe, CdS and CdTe crystals [8-10] have become possible due to the existence of so-called isotropic point at a certain wavelength (the information on the isotropic point see, e.g., in [7]). For example, for $\mathrm{CdS}$ crystals at the liquid-nitrogen temperature and the light wavelength $\lambda=511 \mathrm{~nm}$, the appearance of the gyration tensor component $0.45 \times 10^{-4}$ has been found at the pressure of $0.074 \mathrm{kbar}$. This corresponds to the piezogyration tensor component of the order of $-10^{-12} \mathrm{~m}^{2} \mathrm{~N}^{-1}$ [9]. In GaAs crystals $(T=77 \mathrm{~K}$ and $\lambda=917 \mathrm{~nm})$ [8], the gyration $8.9 \times 10^{-4}$ appears at the pressure of $45 \mathrm{~kg} / \mathrm{mm}^{2}$, thus corresponding to the piezogyration coefficient of $\sim 10^{-11} \mathrm{~m}^{2} \mathrm{~N}^{-1}$ at the wavelength close to the absorption edge. Nonetheless, the conditions of observation of the piezogyration in [8-10] have been quite complicated: low temperature, fixed wavelength of isotropic point, etc. One of the first works devoted to experimental observation of the piezogyration effect induced by directed pressures, against the background of intrinsic and piezo-induced optical birefringences, has been published in 1979 by H.-J. Weber [11]. In this paper the magnitude of the piezogyration tensor component has been determined for $\mathrm{NaClO}_{3}$ crystals $\left(10^{-14}-10^{-15} \mathrm{~m}^{2} \mathrm{~N}^{-1} \quad\right.$ [11]). The piezogyration effect has also been studied in low-symmetry ammonium Rochelle salt 
crystals against the background of linear birefringence [12]. The coefficient $\beta_{3131}$ of the piezogyration tensor has been determined as $\beta_{3131}=(4.6 \pm 0.8) \times 10^{-13} \mathrm{~m}^{2} \mathrm{~N}^{-1}$ for $\lambda=633 \mathrm{~nm}$ and $T=287 \mathrm{~K}[12]$.

The appearance of elastogyration induced by spontaneous deformation in the course of ferroelastic phase transition has been revealed in [13]. It has been shown further on that the piezogyration or elastogyration effects could lead not only to changes in the already existing optical activity. Another result of those effects is a piezo-induced optical activity appearing in crystals, which have remained optically inactive before the application of mechanical stress [14]. Such the crystals should belong to the groups of symmetry $\overline{4} 3 \mathrm{~m}, 6 \mathrm{~mm}, 4 \mathrm{~mm}$ and $3 \mathrm{~m}$. On the basis of symmetry principles of twinning in ferroics, the authors [14] have shown that the ferroelastic domains can be enantiomorphous under the phase transitions governed by the symmetry changes $\overline{4} 3 \mathrm{mF}^{4} 2 \mathrm{~m}, \overline{4} 3 \mathrm{mF} 222, \quad 6 \mathrm{mmFmm} 2,4 \mathrm{mmFmm} 2,6 \mathrm{mmF} 2$ and $4 \mathrm{mmF} 2$, i.e. switching of the domain structure by mechanical stresses should change a sign of the gyration tensor.

Anomalous behaviour of the piezogyration effect in the course of ferroelastic phase transition in $\mathrm{K}_{2} \mathrm{Cd}_{2}\left(\mathrm{SO}_{4}\right)_{3}$ crystals has been revealed in [15]. The coefficients of piezogyration tensor were found to be of order $\sim 10^{-13} \mathrm{~m}^{2} \mathrm{~N}^{-1}$ [15]. It is worth noticing that the piezogyration effect found in all the mentioned cases has proved to be too small for any practical use. The effect has been regarded as interesting from a purely academic viewpoint rather than the viewpoint of practical applications. On the other hand, searching of materials with high figure of merit describing the piezogyration effect is still important, since the effect could be, in principle, used for controlling optical radiation. The present work is devoted to studies of the piezogyration effect in paratellurite crystal, which is one of the most often utilized acoustooptical materials.

\section{Experimental}

We have studied $\mathrm{TeO}_{2}$ crystals which belong to the point group of symmetry 422 . The matrices of both polar and axial tensors of the rank four have the same form for this symmetry group [2], so that we present here only the matrix for the piezogyration tensor:

$\beta_{l k m n}=$\begin{tabular}{r|cccccc} 
& $\sigma_{11}$ & $\sigma_{22}$ & $\sigma_{33}$ & $\sigma_{32}$ & $\sigma_{31}$ & $\sigma_{21}$ \\
\hline$\Delta g_{11}$ & $\beta_{1111}$ & $\beta_{1122}$ & $\beta_{1133}$ & 0 & 0 & 0 \\
$\Delta g_{22}$ & $\beta_{1122}$ & $\beta_{1111}$ & $\beta_{1133}$ & 0 & 0 & 0 \\
$\Delta g_{33}$ & $\beta_{3311}$ & $\beta_{3311}$ & $\beta_{3333}$ & 0 & 0 & 0 \\
$\Delta g_{32}$ & 0 & 0 & 0 & $\beta_{3232}$ & 0 & 0 \\
$\Delta g_{31}$ & 0 & 0 & 0 & 0 & $\beta_{3232}$ & 0 \\
$\Delta g_{21}$ & 0 & 0 & 0 & 0 & 0 & $\beta_{2121}$
\end{tabular}.


This means that, for example, the application of mechanical stress $\sigma_{33}$ yields in the same changes of the optical-frequency impermeability components $B_{11}$ and $B_{22}$ (the same being true of the refractive indices):

$$
B_{11}=B_{22}=B_{11}^{0}+\pi_{1133} \sigma_{33},
$$

where $B_{11}^{0}=1 / n_{0}^{2}$ is the optical-frequency impermeability at zero stress, $n_{0}$ the ordinary refractive index and $\pi_{1133}$ the piezooptic coefficient. Thus, such the external action should remain the crystal optically uniaxial. On the other hand, the optical activity should change its value due to the piezogyration effect:

$$
\Delta g_{33}=\frac{\Delta \rho \lambda n_{0}}{\pi}=\beta_{3333} \sigma_{33},
$$

where $\Delta \rho$ denotes the increment of specific rotation of polarization plane and $\lambda$ the wavelength of optical radiation. When the light propagates along the optic axis and the compressive stress is applied along the same direction, the accompanying linear optical birefringence in the $\mathrm{Z}$ direction does not appear. Then it is possible to study the piezogyration effect via direct measurements of the rotation of polarization plane.

The optical set-up is presented in Fig. 1. Linearly polarized light of He-Ne laser (1) was transformed into circularly polarized one with a quarter-wave plate (2) and then linearly polarized beam was formed with a polarizer (3) installed into rotation stage. The quarter-wave plate (2) and the polarizer (3) were used in order that the linearly polarized light incident on the input surface of sample (4) had the intensity independent of any rotations of the polarizer. The angle of rotation of the light polarization plane was measured with the aid of Faraday cell 5, analyzer 6, photo multiplier 7 and oscilloscope 8.

The sample was inserted into set-up specially designed for application of longitudinal mechanical stresses (see Fig. 2).

The mechanical tension of the sample was caused by the system of arms that pushes the hollow rod, with the hole diameter of $\varnothing 5.0 \mathrm{~mm}$. For making the stress distribution

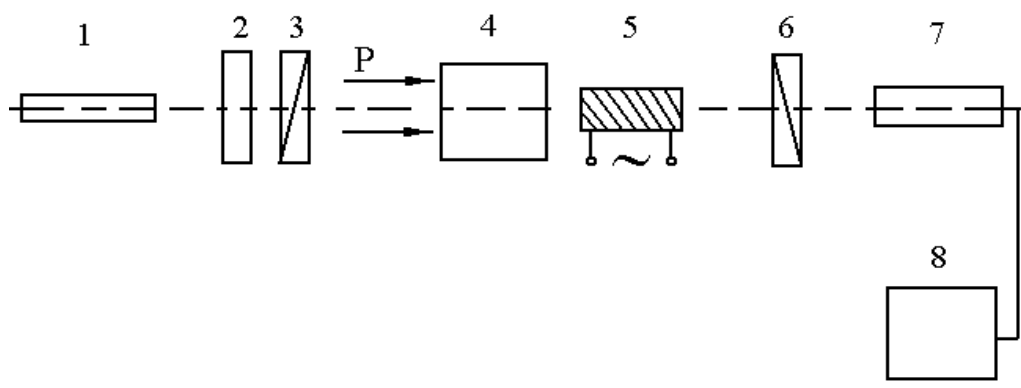

Fig. 1. Polarimetric set-up for studies of the piezogyration effect: 1 - He-Ne laser; 2 -quarter-wave plate; 3 - polarizer installed into rotation stage; 4 - sample inserted into set-up for application of longitudinal mechanical stress; 5 - Faraday cell; 6 - analyzer; 7 - photomultiplier and 8 - oscilloscope. 


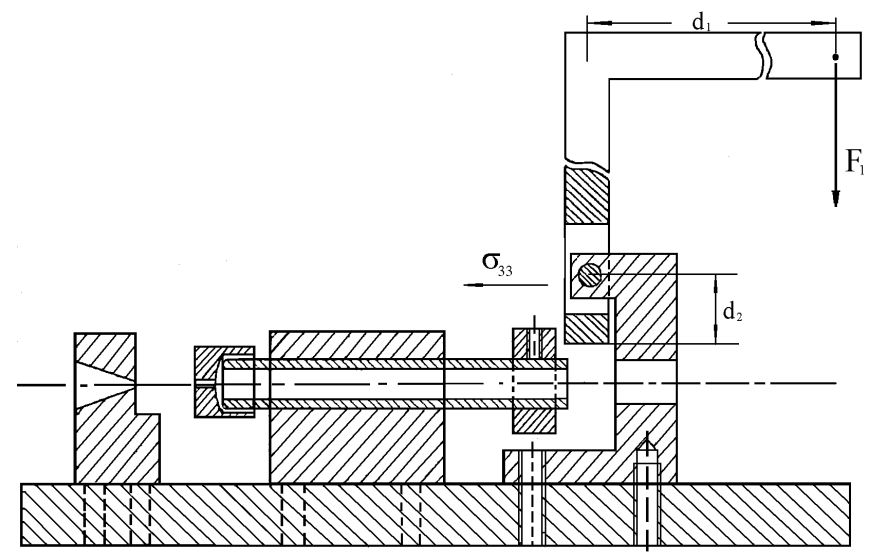

Fig. 2. Set-up for application of longitudinal mechanical stresses.

more homogeneous, the rod was supplied by cricoid tip with a hole $(\varnothing 1.0 \mathrm{~mm})$. On the other side of the sample, the prop with the same hole was mounted on the massive rest. The corresponding relation for determination of compressive stress may be written as

$$
\sigma_{33}=\frac{F_{1} d_{1}}{S d_{2}}
$$

where $F_{1}$ is the force applied to one of the arms, $d_{1}$ and $d_{2}$ the lengths of the arms and $S$ the cross-section square of the sample.

Linearly polarized light propagated through the holes and the sample. The sample of $\mathrm{TeO}_{2}$ crystal with the thickness $d_{z}=5.01 \mathrm{~mm}$ and the cross section square $S=32.87 \mathrm{~mm}^{2}$ was prepared for our experiments.

\section{Experimental results and discussion}

It has been found that the paratellurite crystals are levorotary and the value of specific optical rotation is equal to $\rho=-86 \mathrm{deg} / \mathrm{mm}$ at $\lambda=632.8 \mathrm{~nm}$. This value is in good agreement with that previously obtained in the work [16] $(\rho=-(86.9 \pm 0.5) \mathrm{deg} / \mathrm{mm})$. The compressive mechanical stress leads to increase in the natural optical rotation. For checking whether any "parasitic" linear birefringence is induced by mechanical stress or not, we have performed complementary experiments. Namely, we have measured the rotation angle of the azimuth of polarization ellipse of the emergent light depending on the polarization azimuth of the incident light (see Fig. 3).

As one can see from Fig. 3, essential experimental data scattering takes place in this experiment. Such the scattering might be caused by both high experimental errors and oscillations of polarization azimuth of the emergent light, due to existence of intrinsic and piezo-induced birefringences. Let us use the formula for the oscillations of polarization ellipse derived in [11]:

$$
\Delta \eta=\frac{1}{4 \varphi}\left[1-\frac{\sin 2 \varphi}{2 \varphi}-\frac{\sin ^{2} \varphi}{\varphi} \sin (4 \phi 2 \varphi)\right] \delta \Delta \delta+\Delta \varphi,
$$


where $\varphi=\frac{\pi d_{z} g_{33}}{\lambda n}$ means the angle of optical rotation caused by the natural optical activity, $\Delta \varphi$ the increment of the optical rotation resulted from the piezogyration, $\delta=\frac{2 \pi \Delta n_{l} d_{z}}{\lambda}$ the retardation caused by intrinsic linear birefringence and $\Delta \delta$ the increment of the mentioned retardation related to the piezooptic effect. Basing on the numerical simulation data, one can find that the amplitude of oscillations of the polarization ellipse could achieve the magnitude of 1.5-3.0 deg only if the linear birefringence were of order of $10^{-3}$, while its piezo-induced increment were equal to $10^{-4}$. Notice that both the values are unrealistic. Hence, one can conclude that the scattering of experimental data seen from Fig. 3 is caused purely by experimental errors, which in our case are as large as $34 \%$. Nevertheless, if the linear birefringence really exists in the $\mathrm{Z}$ direction of $\mathrm{TeO}_{2}$ crystals, its influence on the results of our measurements would fall within the range of the experimental errors.

The dependence of increment of the specific polarization ellipse rotation on the mechanical stress is shown in Fig.4. Basing of this linear dependence and Eq. (6), one can calculate the coefficient of piezogyration effect for $\mathrm{TeO}_{2}$ crystal, which is equal to $\beta_{3333}=-(1.5 \pm 0.5) \times 10^{-12} \mathrm{~m}^{2} / \mathrm{N}$. Let us notice that the piezogyration coefficient obtained for $\mathrm{TeO}_{2}$ crystals is, in the order of magnitude, larger than the corresponding coefficient, e.g., for $\mathrm{SiO}_{2}$ crystals. Now we can estimate the value of elastogyration coefficient $\delta_{3333}$, using the equation $\delta_{3333}=2 C_{1133} \beta_{3311}+C_{3333} \beta_{3333}$. Unfortunately, the value of the

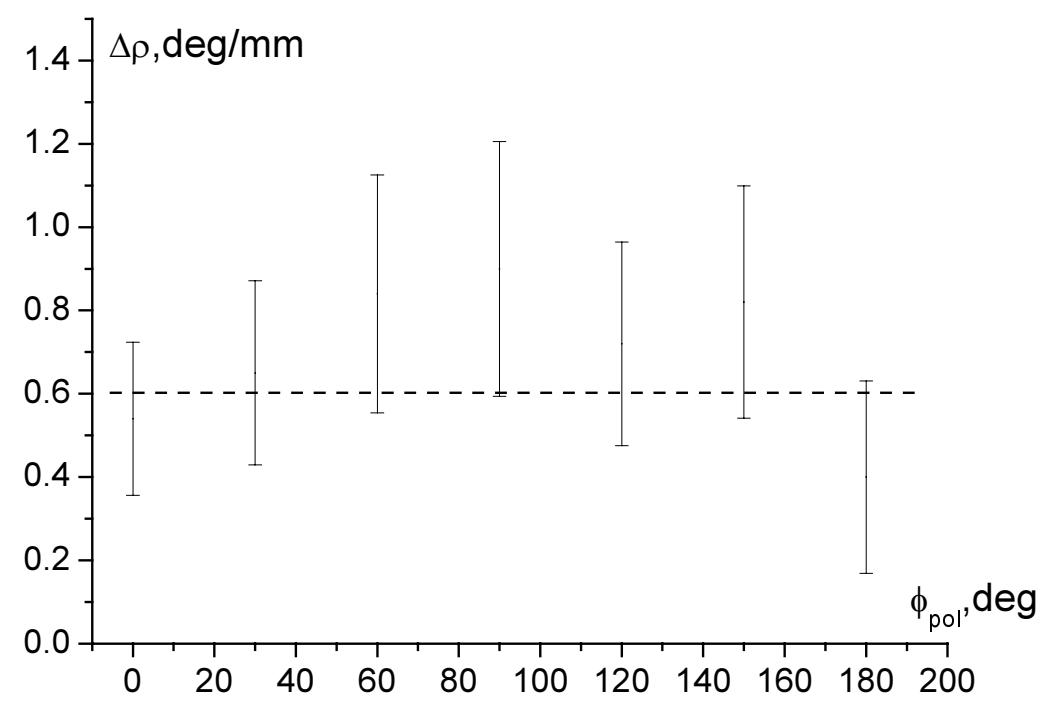

Fig. 3. Dependence of specific angle $\Delta \rho$ of piezo-induced rotation of the polarization azimuth on the orientation angle of polarizer $\phi_{\text {pol }}$ measured for the mechanical stress $\sigma_{33}=3.7 \times 10^{6} \mathrm{~N} / \mathrm{m}^{2}$. 


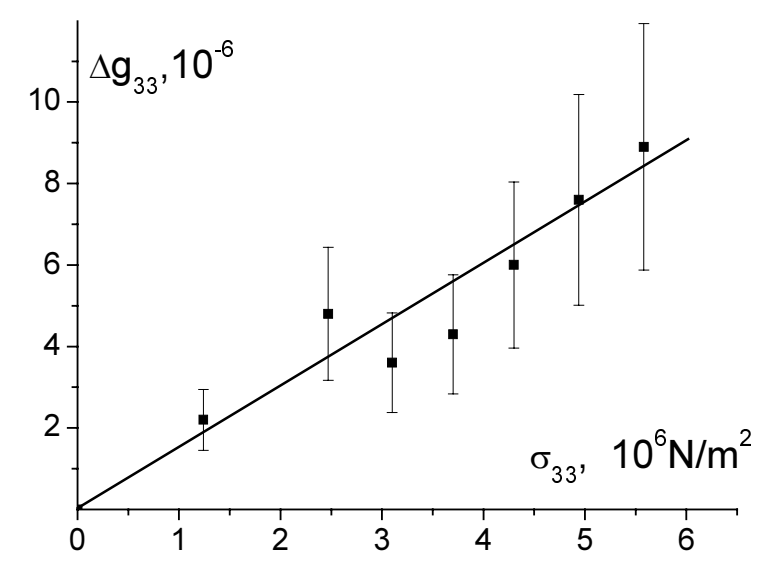

Fig. 4. Dependence of specific rotation of polarization ellipse on mechanical stress obtained for $\mathrm{TeO}_{2}$ crystals at $\lambda=632.8 \mathrm{~nm}$ and $T=293 \mathrm{~K}$.

piezogyration coefficient $\beta_{3311}$ is unknown. However, it is known that $C_{1133}<<C_{3333}$, namely we have $C_{3333}=1.05 \times 10^{11} \mathrm{Nm}^{-2}$ and $C_{1133}=0.2 \times 10^{11} \mathrm{Nm}^{-2}$ [17]. If we neglect the term $C_{1133} \beta_{1133}$, the mentioned formula is reduced to $\delta_{3333} \approx C_{3333} \beta_{3333}$. Then the estimated value of elastogyration coefficient would be equal to $\delta_{3333} \approx-0.2$. Let us now derive the acoustogyration figure of merit defined by the formula [18]

$$
M_{2}^{a g}=\frac{\delta_{3333}^{2}}{\rho v_{33}^{3} n^{2}},
$$

with $\rho=5.99 \times 10^{3} \mathrm{~kg} / \mathrm{m}^{3}$ being the density of $\mathrm{TeO}_{2}$ crystals, $v_{33}=4.3 \times 10^{3} \mathrm{~m} / \mathrm{s}$ the velocity of longitudinal acoustic wave and $n_{o}=2.26$ the refractive index. We obtain the value equal to $1.64 \times 10^{-17} \mathrm{~s}^{3} / \mathrm{kg}$, which is two orders of magnitude larger than that known for the quartz crystals $\left(M_{2}^{a g} \approx 1.6 \times 10^{-19} \mathrm{~s}^{3} / \mathrm{kg}\right)$.

\section{Conclusions}

In this paper we have presented the results of studies of the longitudinal piezogyration effect in $\mathrm{TeO}_{2}$ crystals. The coefficient of the piezogyration effect for these crystals has been defined for the normal conditions and the light wavelength of $\lambda=632.8 \mathrm{~nm}$. It is equal to $\beta_{3333}=-(1.5 \pm 0.5) \times 10^{-12} \mathrm{~m}^{2} / \mathrm{N}$. The estimated values of the elastogyration tensor coefficient and the acoustogyration figure of merit are respectively $\delta_{3333} \approx-0.2$ and $M_{2}^{a g} \approx 1.64 \times 10^{-17} \mathrm{~s}^{3} / \mathrm{kg}$.

\section{References}

1. Aizu K, 1964. Ferroelectric transformations of tensorial properties in regular ferroelectrics. Phys. Rev. 133: A1350-A1359.

2. Vlokh OG and Krushel'nitskaya TD, 1970. Axial fourth-rank tensors and quadratic 
electro-gyration, Kristallogr. 15: 587-589.

3. Lvov VS, 1967. Optical activity of deformed crystals. Fiz. Tverd. Tela. 9: 12731275.

4. Myers MB and Vedam K, 1966. Effect of pressure on the optical rotatory power and dispersion of alpha-quartz . J. Opt. Soc. Amer. 56:1741-1748.

5. Myers MB and Vedam K, 1967. Effect of pressure on the optical rotary power and dispersion of crystalline sodium chlorate. J. Opt. Soc. Amer. 57:1146-1148.

6. Vlokh RO, Pyatak YI and Skab IP, 1991. Piezogyration in enantiomorphous quartz crystals. Opt. Spektrosk. 70: 243-244.

7. Hobden MV, 1968. Optical activity in a non-enantiomorphous crystal: $\mathrm{AgGaS}_{2}$. Acta Cryst. A24: 676-680.

8. Solov'ev LE, 1979. Piezooptical activity of cubic crystals. Opt. Spektrosk. 46: 10201022.

9. Zilbernstein AKh, Kozicyna SYu and Solov'ev LE, 1976. Studies of the phenomenon of pseudo-crossing of dispersion curves under electric field and axial deformations. Opt. Spektrosk. 41:513-515.

10. Solov'ev LE and Chayka MO, 1980. Optical activity of ZnSe, GaAs and CdTe induced by uniaxial deformation. Fiz. Tverd. Tela. 22: 970-975.

11. Weber H-J, 1979. Electrogyration and piezogyration in $\mathrm{NaClO}_{3}$. Acta Cryst. A35: 225-232.

12. Berezhnoy IV, Vlokh OG and Shopa YaI, 1987. Gyrotropic properties of ammonium Rochelle salt. Izv. AN SSSR. Ser. Fiz. 51: 2186-2189.

13. Vlokh OG and Lazko LA, 1984. Optical properties of the $\mathrm{K}_{2} \mathrm{Cd}_{2}\left(\mathrm{SO}_{4}\right)_{3}$ ferroelastic. Ferroelectrics. 56: 1107-1110.

14. Vlokh R O and Slivka V Y, 1989. Enantiomorphism of ferroelastic domains. Ferroelectrics. 98: 167-169.

15. Vlokh RO, Gotra ZYu, Kovpak OV and Skab IP, 1995. Piezogyration in $\mathrm{K}_{2} \mathrm{Cd}_{2}\left(\mathrm{SO}_{4}\right)_{3}$ ferroelastics. Ukr. Fiz. Zhurn. 40: 342-344.

16. Uchida N, 1971. Optical properties of single-crystal paratellurite $\left(\mathrm{TeO}_{2}\right)$. Phys. Rev. B. 4: 3736-3745.

17. Shaskolskaya MP Acoustic crystals. Moscow: Nauka (1982).

18. Vlokh R, Krupych $\mathrm{O}$ and Martynyuk-Lototska I, 2007. On the problem of acoustogyration diffraction of light. Ukr. J. Phys. Opt. 8: 143-157. 